\title{
The effect of retrieval focus and emotional valence on the inferior frontal cortex activity during autobiographical recollection
}

\author{
Ekaterina Denkova ${ }^{1}$ *, Sanda Dolcos ${ }^{2}$ and Florin Dolcos ${ }^{2,3,4}$ * \\ ${ }^{1}$ Alberta Cognitive Neuroscience Group, University of Alberta, Edmonton, AB, Canada \\ ${ }^{2}$ Psychology Department, University of Illinois at Urbana-Champaign, Urbana, IL, USA \\ ${ }^{3}$ Neuroscience Program, University of Illinois at Urbana-Champaign, Urbana, IL, USA \\ ${ }^{4}$ Beckman Institute for Advanced Science and Technology, University of Illinois at Urbana-Champaign, Urbana, IL, USA
}

\section{Edited by:}

Hans J. Markowitsch, University of

Bielefeld, Germany

\section{Reviewed by:}

Lucina Q. Uddin, Stanford University, USA

Alain Morin, Mount Royal University, Canada

\section{*Correspondence:}

Ekaterina Denkova and Florin Dolcos, Social, Cognitive, Personality, and Emotional (SCOPE) Neuroscience Lab, Psychology Department, Neuroscience Program, Beckman Institute for Advanced Science and Technology, University of Illinois at Urbana-Champaign, 405 North Mathews Avenue, Room 2057, Urbana-Champaign, IL 61801, USA e-mail: ekaterina.denkova@yahoo.ca; fdolcos@illinois.edu
Although available evidence points to a role of the inferior frontal cortex (IFC) in both emotion processing and autobiographical memory (AM) recollection, it is unclear what the role of this region is in emotional AM recollection. The present study investigated whether IFC activity can be influenced by manipulations of the retrieval focus (emotional vs. non-emotional) and whether this influence is similar for AMs with positive and negative emotional valence. Participants were asked to focus either on emotional (Emotion condition) or on non-emotional contextual (Context condition) details during the elaboration of positive and negative AMs, while fMRI data were collected. The study yielded two main findings: (1) Focusing on Emotion compared to Context during AM recollection was associated with increased activity in bilateral IFC, for positive AMs, whereas negative AMs produced similarly high IFC activity during Emotion and Context conditions; (2) There was a hemispheric dissociation in the IFC linked to the experiencing of emotion and the focus of AM recollection, such that the left IFC activity correlated positively with the subjective re-experience of emotion during the Emotion condition, whereas the right IFC activity correlated negatively with the subjective re-experience of emotion during the Context condition, for both positive and negative AMs. Overall, the present findings suggest that IFC's involvement during the recollection of emotional AMs is susceptible to manipulations of the retrieval focus only in the case of positive AMs, and that this region plays a role in both the enhancement and inhibition of emotional experience during AM recollection.

Keywords: episodic memory, emotional valence, retrieval goal, inferior frontal gyrus, insula

\section{INTRODUCTION}

There is considerable evidence that the inferior frontal cortex (IFC) plays an important role in language (Poldrack et al., 1999), cognitive control (Badre, 2008), memory (Thompson-Schill et al., 1997; Fletcher and Henson, 2001), emotion processing (Wager et al., 2008; Lindquist et al., 2012), and emotion regulation (Ochsner et al., 2012), possibly through its involvement in operations such as language unification (Hagoort, 2005), controlled retrieval (Badre and Wagner, 2007), selection among competing alternatives (Thompson-Schill et al., 1999; Moss et al., 2005; Grindrod et al., 2008), integration of information (Fuster, 2002), and response inhibition (Aron et al., 2004). Of particular interest for the present investigation, evidence derived from separate lines of research points to a pivotal role of the IFC in memory retrieval (Greenberg et al., 2005; Badre and Wagner, 2007) and in emotion processing (Wager et al., 2008; Lindquist et al., 2012). The goal of the present investigation is to elucidate the role of IFC in the retrieval of emotional autobiographical memories (AMs) according to the retrieval focus and the valence of memories.

Functional neuroimaging evidence from separate lines of investigations suggests a role of the IFC in both the enhancement and inhibition of emotion processing (Hooker and Knight, 2006; Dolcos et al., 2011; Ochsner et al., 2012; Iordan et al., 2013). Studies investigating the influence of emotion on memory have provided evidence for a role of the IFC in enhancing the effect of emotion on memory formation (Dolcos et al., 2004) and in diminishing the impact of negative goal-irrelevant emotional distraction on working memory (Dolcos et al., 2006, 2008, 2013). Studies investigating the neural correlates of emotion control have pointed to the IFC's contribution to voluntary up- and down-regulation of emotion (Ochsner et al., 2004; Kim and Hamann, 2007). The evidence from these investigations is based nearly exclusively on externally triggered emotions in experimental settings (e.g., by viewing emotional pictures), most of the time negative, rather than on internally triggered emotions (e.g., recall of emotional personal memories). Here, we investigate the role of IFC in the processing of internally triggered emotions associated with the recollection of emotional AMs.

Internally triggered emotions are more ecologically valid and can generate stronger emotional responses than those produced by external stimulation in experimental settings (Salas et al., 2012). Also, the former are at the basis of maintaining affective disorders, 
such as depression and post-traumatic stress disorder (Brewin et al., 1999; Rubin et al., 2008), which are characterized by an increased focus toward negative personal memories and/or inhibition of positive ones (Werner-Seidler and Moulds, 2011). Recollection of emotional AMs has been linked, among other brain regions, to the involvement of IFC (Markowitsch et al., 2000). The more ventral portion of the IFC, part of the temporo-frontal junction interconnected through the ventral branch of the uncinate fascicle, has been attributed a crucial role in "synchronizing emotional and factual components of the personal past" during remembering (Brand and Markowitsch, 2008; p. 326; Markowitsch, 1995; Brand and Markowitsch, 2006). IFC's involvement has also been found in "non-emotional" AM studies (Conway et al., 1999; Piolino et al., 2004; Greenberg et al., 2005; Daselaar et al., 2008). Typically, this region has been associated with successful memory retrieval, which involves strategic search and selection of appropriate information and monitoring of the veracity and cohesiveness of the recollected memory (Svoboda et al., 2006; Badre and Wagner, 2007). It is not clear, however, whether IFC's involvement during the recollection of emotional AMs can be influenced by the focus of retrieval (emotional vs. non-emotional), and whether this influence is similar for positive and negative AMs. Given that positive and negative AMs may be governed by different mechanisms and lead to different outcomes (Denkova et al., 2012), and that IFC appears to be more involved for negative AMs (Markowitsch et al., 2003), it is important to clarify the role of emotional valence in the retrieval of emotional AMs. While negative memories have received, overall, much more attention in the literature, there is also evidence highlighting the importance of positive memories in promoting personal self-esteem and overall positive mindset (Diener and Seligman, 2002; Fredrickson, 2004; D’Argembeau and Van der Linden, 2008; Denkova et al., 2012), and their beneficial effects in depressed people (Dalgleish et al., 2013).

The main goal of the present study was to investigate the involvement of the IFC during the recollection of emotional AMs, linked to the focus of retrieval (emotional vs. non-emotional) and the valence of memories (positive vs. negative). For this purpose, fMRI data were recorded while participants were cued to focus either on emotional (Emotion condition) or on nonemotional contextual (Context condition) details, during elaboration of highly emotional positive and negative AMs. Based on the extant evidence, we made the following predictions. General sensitivity of the IFC to manipulations of the retrieval focus should be reflected in differential engagement of this region in the Emotion and Context conditions, for both positive and negative AMs, such that increased IFC activity during the Emotion condition would be associated with enhanced emotional experience. However, it is also expected that retrieval focus may also result in specific sensitivity of the IFC response linked to the valence of AMs, possibly reflecting the enhancement of positive emotions and the inhibition of negative emotions.

\section{MATERIALS AND METHODS PARTICIPANTS}

Analyses were performed on data from 17 right-handed native English speaking young adults (6 men; age range 18-46, mean $=26.06$ years, $\mathrm{SD}=7.20)$, who provided written informed consent and received payment for their participation. The experimental protocol was approved by the Institutional Health Research Ethics Board.

\section{COLLECTION AND SELECTION OF EMOTIONAL AUTOBIOGRAPHICAL MEMORIES}

Personal memories were elicited from each participant during an interview performed approximately 5 weeks prior to the fMRI session using an autobiographical memory questionnaire (AMQ) (Denkova et al., 2012). The AMQ comprised a list of 115 verbal cues for distinct life events (e.g., "the birth of a family member," "being hospitalized"); for each of them, participants were asked to remember a unique episode from their life and to provide a brief description of the memory, which was then used as a personalized memory cue during the fMRI scanning. Phenomenological characteristics of each event were assessed by asking the participants to date the memory and rate it on several Likert scales including Emotional Valence (using a 7 -point scale: $-3=$ very negative, $0=$ neutral, and $+3=$ very positive), Emotional Intensity, Personal Significance, Vividness, the amount of Contextual Details, and the Frequency of Retrieval (all of the latter used a 7 -point scale: $1=$ not at all, $7=$ extremely). For each participant, we selected the 40 most emotional memories (20 positive and 20 negative), based on the emotional ratings. Half of the selected memories, with an equal proportion of positive and negative, were assigned to the Emotion condition, and the other half of AMs were assigned to the Context condition.

\section{fMRI TASKS}

\section{The autobiographical memory tasks}

(i) In the Emotion focus condition, participants were instructed to remember the specific event and focus on the emotional aspects of their memories, including associated sensations and feelings (e.g., butterflies in the stomach, palpitations). (ii) In the Context focus condition, participants were instructed to remember the specific event and focus on the contextual aspects of their memories, by retrieving as many contextual details as possible (e.g., about where and when the event occurred). Each memory cue was preceded either by the instruction cue "Remember Emotion" (for the Emotion condition), or "Remember Context" (for the Context condition). After recollection, each event was rated on three fivepoint Likert scales including Emotional Intensity, Vividness, and Reliving ( $1=$ very low; $5=$ very high $)$.

\section{The semantic memory control task}

In line with other AM studies (Greenberg et al., 2005; Young et al., 2013), we also used a control condition involving semantic memory (SM) retrieval, such as the generation of exemplars from different semantic categories (e.g., sports, vegetables) (Battig and Montague, 1969). Each semantic category cue was preceded by the instruction cue "Generate Examples." To be consistent with AM conditions, each exemplar generation was rated on three fivepoint Likert scales including Vividness, Difficulty of the task, and approximate Number of the recalled items.

\section{fMRI DESIGN}

The AM and SM conditions had the same general structure (Denkova et al., 2011). Each trial began with an instruction screen 
for $2 \mathrm{~s}$, immediately followed by a memory cue for $4 \mathrm{~s}$. After the cue offset, a fixation screen was presented for $10 \mathrm{~s}$ during which participants elaborated their personal memories or generated exemplars. The end of the retrieval period was marked by an instruction screen for upcoming ratings, for $1.5 \mathrm{~s}$. Then, each of the three ratings was presented for $2.5 \mathrm{~s}$ and in a counterbalanced order across trials. The ratings were followed by an inter-trial interval of variable duration $(2-9 \mathrm{~s}$, average $=6 \mathrm{~s})$, before the beginning of the next trial.

\section{MRI DATA COLLECTION}

MRI data were recorded using a 1.5-T Siemens Sonata scanner. The anatomical images were 3D MPRAGE anatomical series [repetition time $(\mathrm{TR})=1600 \mathrm{~ms}$, echo time $(\mathrm{TE})=3.82 \mathrm{~ms}$, field of view $(\mathrm{FOV})=256 \mathrm{~mm} \times 256 \mathrm{~mm}$, number of slices $=112$, voxel size $=1 \mathrm{~mm} \times 1 \mathrm{~mm} \times 1 \mathrm{~mm}]$. The functional images consisted of series of images acquired axially using an echoplanar sequence ( $\mathrm{TR}=2000 \mathrm{~ms}, \mathrm{TE}=40 \mathrm{~ms}, \mathrm{FOV}=256 \mathrm{~mm} \times 256 \mathrm{~mm}$, number of slices $=28$, voxel size $=4 \mathrm{~mm} \times 4 \mathrm{~mm} \times 4 \mathrm{~mm}$ ).

\section{fMRI DATA ANALYSIS}

Statistical analyses, performed with SPM2 (Statistical Parametric Mapping), were preceded by the following pre-processing steps: Quality Assurance, TR Alignment, Motion Correction, Coregistration, Normalization, and Smoothing $(8 \mathrm{~mm}$ full-width half maximum isotropic Kernel). At the individual level, each event was modeled by the canonical hemodynamic response function ( $h r f$ ) and its temporal derivate. The $h r f$ was time-locked to 2 s (1 TR) following the onset of the memory cues, in the Emotion and Context AM conditions, and $1 \mathrm{~s}$ ( $0.5 \mathrm{TR})$ after the onset of the category cue, in the SM condition to allow time for reading the cues. This procedure was guided by the present RT data, which showed that the recognition of the AM cue and beginning of retrieval occurred at an average RT of $1.67 \mathrm{~s}( \pm 0.44)$, and the beginning of the exemplar generation in the SM condition occurred at an average RT of $1.03 \mathrm{~s}( \pm 0.40)$. This procedure allows comparisons of the fMRI signal associated with AM and SM retrieval, by accounting for differences in the timing of retrieval operations and memory identification, and is consistent with the procedure used in previous neuroimaging studies of AM retrieval (Addis et al., 2007). Individual contrasts were computed directly between the different AM event types (e.g., Emotion Positive vs. Context Positive, Emotion Negative vs. Context Negative). These individual contrasts were then entered into group-level $t$ tests, to perform random-effects analyses.

The effects of retrieval focus were investigated by comparing AMs with Emotion focus and AMs with Context focus separately for positive and negative AMs. The interaction effects of retrieval focus and valence were investigated using paired $t$ tests [e.g., (Emotion Positive vs. Context Positive) vs. (Emotion Negative vs. Context Negative)], whose outputs were inclusively masked with the direct contrast of interest (e.g., Emotion Positive vs. Context Positive), to ensure that the interaction difference is due to an existing increased difference in the contrasts of interest. Finally, to investigate whether activity in IFC according to the retrieval focus and valence is linked to the self-reported re-experience of emotion, linear regression analyses were performed between overall IFC activity in each AM condition (i.e., Emotion Positive vs. baseline; Emotion Negative vs. baseline; Context Positive vs. baseline and Context Negative vs. baseline) and the corresponding self-reported emotional ratings.

Activity in regions of interest was investigated using adapted anatomical masks from the Wake Forest University Pick Atlas toolbox. The threshold was set up at $p<0.001$ for the direct contrasts and at $p<0.05$ for the interactions and correlations; the extent threshold was of five contiguous voxels in all analyses. The interaction maps were masked inclusively with the corresponding direct

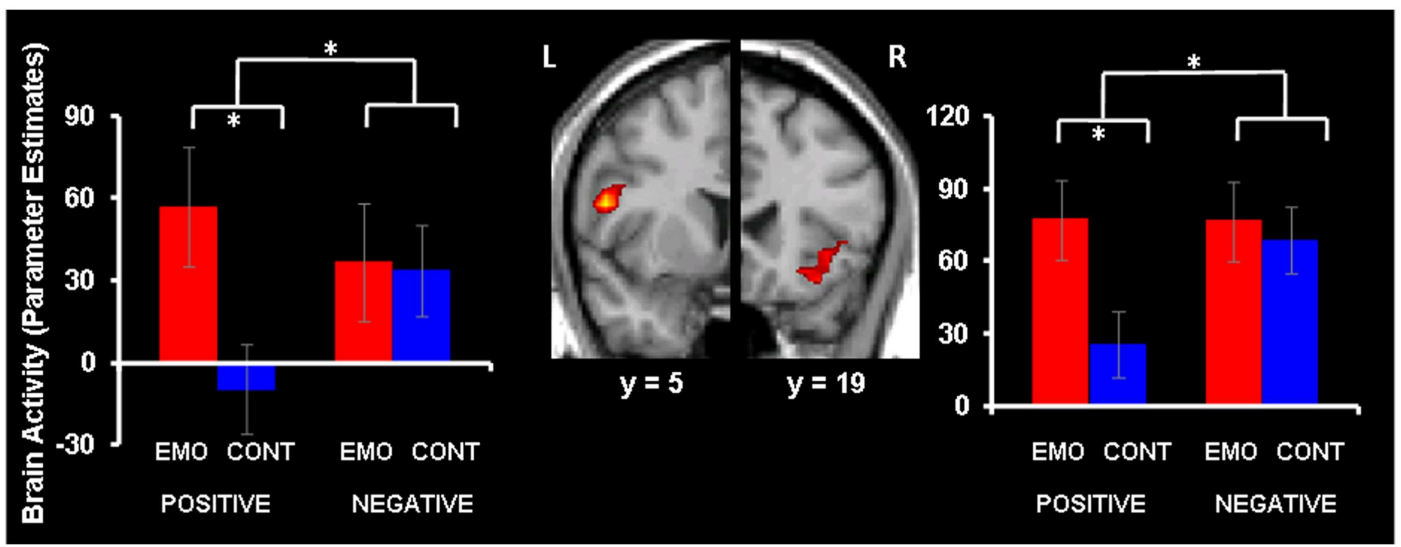

FIGURE 1 | Increased IFC activity for emotion compared to context focus for positive AMs. Focusing on Emotion (EMO) compared to focusing on Context (CONT) led to increased activity in bilateral IFC (red blobs) for positive AMs, whereas negative AMs produced similar IFC activity during EMO and CONT conditions. The interaction map is superimposed on a high resolution brain image displayed in a coronal view (with $y$ indicating the Talairach coordinates on the anterior-posterior axis of the brain). For illustration purpose, the interaction map set up at $p<0.05$ is inclusively masked with the direct contrast set up at $p<0.05$; the effects are also observed when the mask is set up at $p<0.001$ (Table 1). The bar graphs represent the contrast estimates extracted from representative voxels in the left and right IFC, respectively. The error bars correspond to the standard errors of the means. L, Left; R, Right. 
contrast set up at $p<0.001$. Activations in other brain regions, including basic emotion and memory-related medial temporal lobe (MTL) brain areas are reported in a previous report (Denkova et al., 2013). In short, these findings revealed increased activity for positive AMs in the amygdala (AMY) and hippocampus, and in other brain regions, including lateral temporal and prefrontal cortices. Given the similarity of patterns observed in the AMY and IFC, we further investigated the relationship between activity in these two regions, by performing linear regression analyses between IFC activity for Emotion vs. Context contrast (extracted from peak voxels showing significant differences in activation, in the right and left IFC, BA 47) and brain activity in the AMY.

\section{RESULTS}

\section{BEHAVIORAL RESULTS}

Increased re-experiencing of emotion during emotion focused retrieval for both positive and negative AMs

Repeated-measures ANOVA revealed a focus $\times$ ratings interaction $\left[F_{(1,16)}=4.12, p=0.03\right]$, driven by an increase only for the emotional intensity ratings of AMs retrieved with an emotional focus and the absence of significant differences in the other ratings (Reliving and Vividness ratings). The increase was significant for both positive (3.21 vs. $3.03, p=0.02)$ and negative (3.38 vs. 3.07 , $p=0.003)$ AMs.

\section{fMRI RESULTS \\ Increased IFC activity for emotion compared to context focus for positive AMs}

Focusing on Emotion compared to Context led to increased activity in bilateral IFC (BAs 44 and 47) for positive memories, but similarly high IFC engagement under the Emotion and Context foci was observed for negative AMs (see Figure 1 and Table 1). These effects were confirmed by a repeated-measures ANOVA performed on the extracted signal, which, in the left IFC (BA 44), revealed a significant valence $\times$ focus interaction $\left[F_{(1,16)}=15.93, p=0.001\right]$. This interaction was driven by a significant increase in the Emotion compared to the Context condition, for positive $(p<0.001)$ but not for negative $(p=0.80)$ AMs. Similarly, the effect in the right IFC (BA 47) was confirmed by a repeated-measures ANOVA revealing a significant valence $\times$ focus interaction $\left[F_{(1,16)}=7.26, p=0.015\right]$, which was driven by a significant increase in the Emotion compared to the Context condition for positive $(p<0.001)$ but not for negative $(p=0.50)$ AMs.

\section{Hemispheric dissociation in the IFC linked to the experiencing of emotion and the focus of $A M$ recollection}

Brain-behavior correlation analyses revealed opposing patterns of co-variation between activity in the left (showing positive co-variation) and right (showing negative co-variation) IFC and emotional ratings, for Emotion and Context focus, respectively (Figure 2 and Table 1). These effects were common for both positive and negative AMs, as revealed by the conjunction analyses of Emotion Positive $\cap$ Emotion Negative and of Context Positive $\cap$ Context Negative conditions, respectively. Specifically, activity in a left IFC (BA 47) area, extending to the insula, positively
Table 1 | Significant activations and correlations linked to the retrieval focus and the emotional valence of memories.

\begin{tabular}{llll} 
IFC (BA) Side & \multicolumn{2}{l}{ Talairach coordinates } \\
\cline { 2 - 3 }$\quad y$ &
\end{tabular}

\section{DIRECT CONTRASTS}

Emotion Positive vs. Context Positive

$\begin{array}{llrrrrr}\text { IFG (44) } & \text { L } & -55 & 12 & 10 & 4,91 & 8 \\ \text { IFG (47) } & \mathrm{R} & 40 & 27 & -1 & 4,90 & 22 \\ \text { IFG (47) } & \mathrm{L} & -36 & 19 & -8 & 4,85 & 28\end{array}$

\section{INTERACTIONS}

(Emotion Positive vs. Context Positive) vs. (Emotion Negative

vs. Context Negative)

\begin{tabular}{|c|c|c|c|c|c|}
\hline IFG (44) & $\mathrm{L}$ & -55 & 12 & 14 & 3,99 \\
\hline IFG (47) & $\mathrm{R}$ & 32 & 23 & -15 & 2,69 \\
\hline
\end{tabular}

CORRELATIONS

A. Positive correlations

Emotion (Emotion Positive $\cap$ Emotion Negative)

IFG (47) $L \quad-32 \quad 15 \quad-11 \quad 3,13 / 2,45 \quad 7$

IFG (46) L $\quad-48 \quad 28 \quad 17 \quad 2,14 / 2,07$

Context (Context Positive $\cap$ Context Negative)

$\begin{array}{llllll}\text { IFG (46) } L & -51 & 32 & 9 & 2,61 / 2,04 & 12\end{array}$

B. Negative correlations

Context (Context Positive $\cap$ Context Negative)

$\begin{array}{lllllll}\text { IFG (47) } & R & 24 & 35 & -8 & 3,42 / 2,01 & 5\end{array}$

Significant activations and correlations resulting from $\mathrm{ROI}$ analyses are reported. For direct contrasts, a threshold of $p<0.001$ was used. For interactions, a threshold of $p<0.05$ was used and further inclusively masked with the corresponding direct contrast set up at $p<0.001$. For correlations, a threshold of $p<0.05$ was used, and the overlaps between positive and negative AMs within each focus are presented. A cluster size of five contiguous voxels was used for all analyses. IFG, inferior frontal gyrus; BA, Brodmann's area; L, Left, $R=$ Right.

correlated with self-reported re-experience of emotion, for both positive and negative AMs, for the Emotion but not for the Context condition. Similar effects were observed in a more dorsal left IFC (BA 46) area, but they were not specific to the Emotion condition (Table 1). On the other hand, activity in a right IFC (BA 47) area negatively correlated with self-reported re-experience of emotion, for both positive and negative AMs, for the Context but not for the Emotion condition. Overall, these findings suggest a hemispheric dissociation in the IFC linked to the experiencing of emotion and the focus of AM recollection, with the left IFC (BA 47) activity showing specific positive correlations with the subjective re-experience of emotion during the Emotion condition, and the right IFC (BA 47) activity showing specific negative correlations with the subjective re-experience of emotion during the Context condition.

Further analyses revealed positive co-variations between activity in the IFC and the AMY (Figure 3). For positive AMs, positive co-variations were observed between activity in the right IFC and the right $(x=24, y=3, z=-14 ; R=0.67, p=0.002)$ and left $(x=-32, y=-8, z=-13 ; R=0.79, p<0.001)$ AMY, as well as between activity in the left IFC and the right $(x=32, y=-1$, 


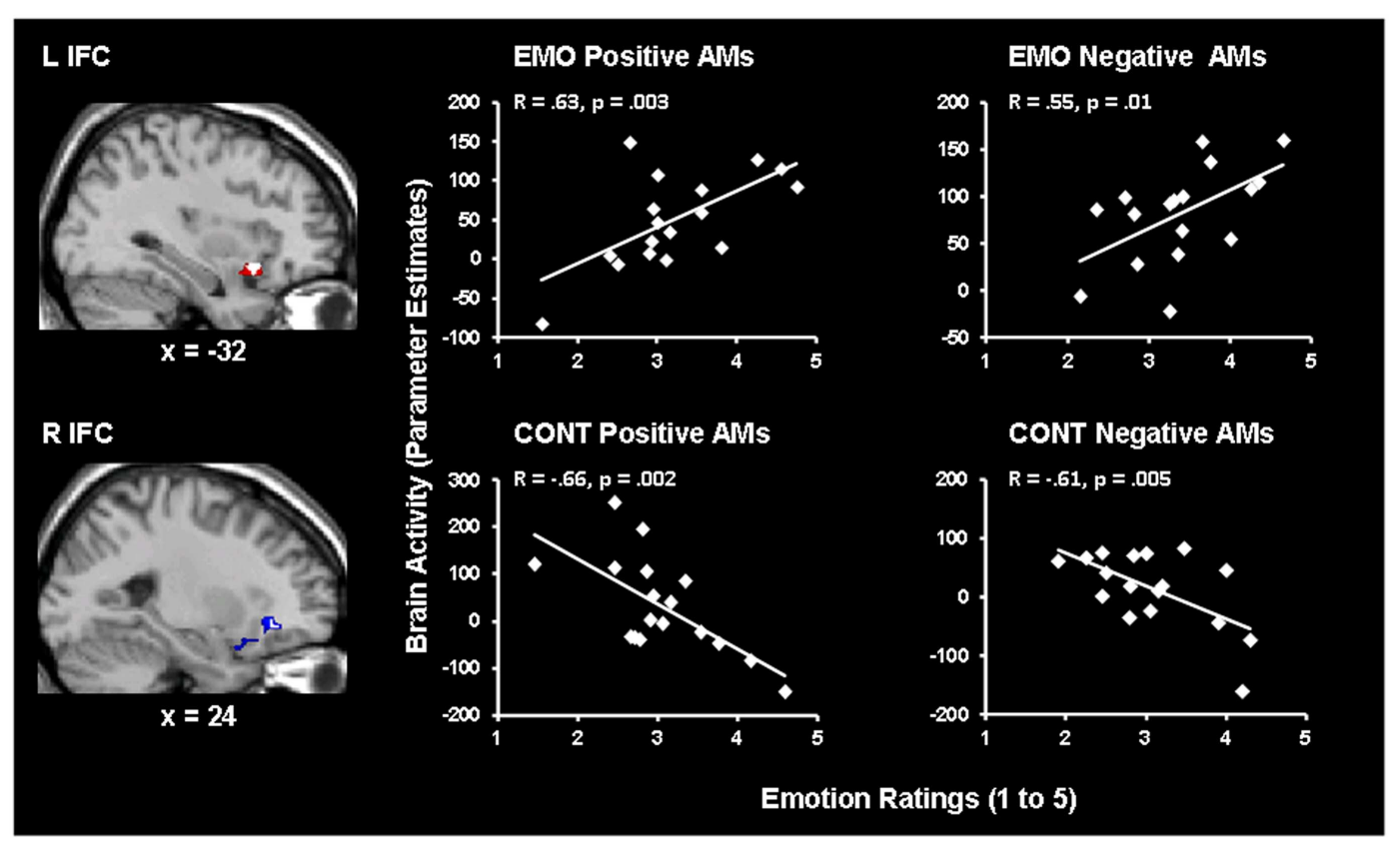

FIGURE 2 | Hemispheric dissociation in the IFC linked to the experiencing of emotion and the focus of $A M$ recollection. Activity in the left IFC correlated positively with emotional ratings in the Emotion (EMO) condition, for both positive and negative AMs, whereas activity in the right IFC correlated negatively with emotional ratings in the Context (CONT) focus, for both positive and negative AMs. The correlation maps in the left and right IFC are superimposed on high resolution brain images displayed in sagittal views (with $x$ indicating the
Talairach coordinates for the left/right hemispheres of the brain). The white blobs represent the areas where there are overlapping voxels for both positive and negative AMs, which are superimposed on larger areas showing positive (red) or negative (blue) co-variations either for positive or for negative AMs. The scatterplots are based on contrast estimates of the IFC activity for each condition, as extracted from the peak voxels of the areas showing the co-variation with the corresponding emotional ratings. L, Left; R, Right. $z=-17 ; R=0.56, p=0.01)$ and left $(x=-28, y=-8, z=-13$; $R=0.66, p=0.002$ ) AMY. Of note, portions of the AMY showing positive co-variation with the IFC overlapped with portions of the AMY areas showing significant increase in activity for positive memories with Emotion vs. Context focus reported in Denkova et al. (2013) (Figure 3). Interestingly, similar patterns of positive co-variations between activity in the IFC and AMY were also observed for negative memories, despite the absence of significant differences in the IFC activity between Emotion and Context. Namely, activity in the right IFC positively correlated with activity in the right $(x=20, y=-1, z=-10 ; R=0.87, p<0.001)$ and left $(x=-32, y=-4, z=-10 ; R=0.78, p=0.001)$ AMY, and activity in the left IFC positively correlated with activity in the right $(x=20, y=-1, z=-10 ; R=0.82, p<0.001)$ and left $(x=-32, y=-8, z=-13 ; R=0.69, p=0.001)$ AMY. An overlap between the correlation and activation patterns was observed only in the left AMY (see Figure 3), given that significant differences in activity between Emotion and Context for negative AMs was revealed only in the left AMY (Denkova et al., 2013).

\section{DISCUSSION}

The present study investigated the IFC's involvement during AM recollection, as a function of the retrieval focus and emotional valence. There were two main findings, which are discussed in turn below.

\section{INCREASED IFC ACTIVITY FOR EMOTION COMPARED TO CONTEXT FOCUS FOR POSITIVE AMs}

This finding is overall consistent with previous investigations linking IFC's involvement to enhanced encoding of emotional items (Dolcos et al., 2004), retrieval of emotional AMs (Markowitsch et al., 2000), as well as voluntary up-regulation of positive emotions (Kim and Hamann, 2007). Importantly, the present finding extends the available evidence by showing that activity in the IFC is susceptible to manipulations of the retrieval focus only during the recollection of positive AMs, showing increased activity during retrieval of positive AMs with an emotion focus and decreased activity when the focus is on other non-emotional contextual details. Keeping in mind that IFC is a heterogeneous structure with distinct subregions (Petrides and Pandya, 2002), the increased activity in different IFC subregions in the present study could be interpreted as follows. Given its role in the subjective experience of emotion (Wager et al., 2008), increased activity in BA 47 could reflect the integration and enhancement of emotional experience during autobiographical retrieval. This interpretation is further supported by the positive relationship between activity in the IFC and the AMY, which suggests that IFC's involvement reflects the integration of emotional information triggered by the AMY and further enhancement of the emotional experience during remembering of AMs with Emotion focus. Given its general role in language production and in inner speech (McGuire et al., 1996; Baciu et al., 1999), particularly during self-referential activities 


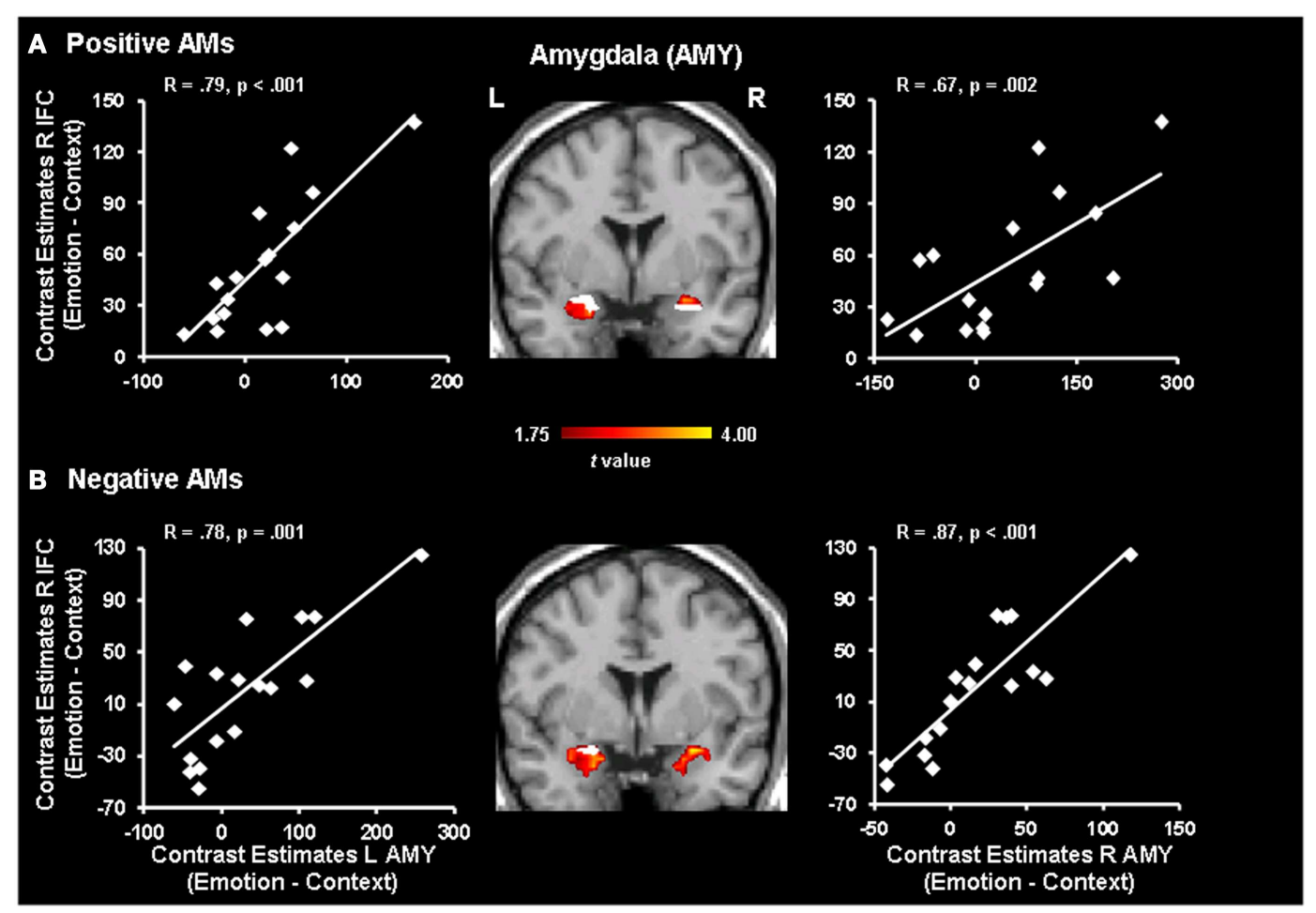

FIGURE 3 | Positive correlations between activity in the IFC and the AMY. Activity in the right Inferior Frontal Cortex (IFC) positively correlated with activity in the left and right AMY (red blobs) for positive (A) and negative (B) AMs, despite the absence of significant differences in the IFC activity between Emotion and Context for the latter. Portions of the AMY showing positive co-variations with the IFC also overlapped (white blobs) with AMY areas showing significant increase in activity for Emotion vs. Context focus. The gradient color bar starts at $p<0.05(t=1.75)$. Similar patterns of correlations were observed in the left IFC (not shown, see text). The scatterplots are based on contrast estimates for Emotion vs. Context extracted from the peak voxel of the areas showing the co-variation with AMY activity. $L$, left; $R$, right.
(Morin and Michaud, 2007), of which autobiographical remembering is an essential part, increased activity in the left BA 44 could be linked to a more general reliance on covert speech mechanisms during recollection of positive AMs with Emotion focus.

The present findings suggest that, compared to the emotion focused positive recollections, context focused positive recollections appear to easily lose their emotionality in the absence of an explicit focus on the (re)experienced emotions. This provides a possible explanation for evidence showing that recalling positive memories cannot always reverse negative mood in depressed people (Joormann et al., 2007), and is consistent with recent evidence from clinical studies suggesting that positive memories could potentially alleviate negative mood depending on the way they are processed (Werner-Seidler and Moulds, 2012; Dalgleish et al., 2013). This finding is also consistent with evidence from healthy participants showing that, unlike negative AMs whose retrieval has a strong direct effect on the post-retrieval negative emotional state, retrieval of positive AMs has a weaker and indirect effect on the positive state (Denkova et al., 2012).

In contrast with positive AMs, remembering negative AMs showed similarly high IFC involvement, regardless of the focus of retrieval. This could reflect the engagement of selection and inhibitory operations, necessary to evaluate the relevance of negative emotional information according to the current retrieval goals, and to incorporate and enhance it when relevant (i.e.,
Emotion focus) and diminish it when not relevant (i.e., Context focus) (Depue et al., 2007). Given the similarity with the response observed in AMY and IFC and their positive co-variation, it is possible that the involvement of some IFC areas reflects the enhancement of the emotional (re)experiencing during the Emotion focus, for both positive and negative AMs, and reduced engagement for positive AMs along with active inhibition of the emotional information (possibly automatically initiated by the AMY) for negative AMs during the Context focus. Overall, these findings suggest that positive AMs can trigger a strong emotional response only if there is an explicit emphasis on the re-experiencing of emotion during remembering, and that this occurs in relationship with activity in the AMY.

\section{HEMISPHERIC DISSOCIATION IN THE IFC LINKED TO THE EXPERIENCING OF EMOTION AND THE FOCUS OF AM RECOLLECTION}

These findings suggest a role of the IFC in the modulation (both enhancement and reduction) of emotional experience. The lateralization of these effects could be interpreted in line with previous evidence linking the left frontal cortex to the retrieval of emotional knowledge during up-regulation of emotional response and the right frontal cortex to inhibitory processes during downregulation of emotional response (Ochsner et al., 2004; Kim and Hamann, 2007). It should be noted that the positive relationship in the left IFC extends to the anterior ventral insula, which is typically 
co-activated with the IFC (Uddin et al., 2013) and is linked to emotion processing (Chang et al., 2012; Kelly et al., 2012; Lindquist et al., 2012), particularly to the awareness of emotional experiences (Craig, 2009; Zaki et al., 2012). Given that in the Emotion AM condition participants were explicitly instructed to focus on emotional details, including the associated sensations and feelings (e.g., butterflies in the stomach, palpitations), the present findings are consistent with a possible contribution of the Anterior Insula together with the IFC to the enhanced emotional experience during Emotion focus, as reflected in the post-retrieval emotional ratings.

Considering altogether the present findings, it could be speculated that the IFC's role in enhancing and inhibiting emotion processing may be linked to its more general involvement according to the relevance of processed information to the current goal (Beer et al., 2006). Specifically, when the emotional information is relevant to the current goal (i.e., retrieval focus on emotional details), it can benefit from enhanced processing through the involvement of IFC, whereas when emotional information is not relevant to the current goal (i.e., retrieval focus on non-emotional contextual details), it can be attenuated or inhibited (Conway and Pleydell-Pearce, 2000; Levine and Edelstein, 2009). Finally, the present brain imaging findings, along with the behavioral findings showing an overall reduction of experienced emotion during the Context focus, could also be linked to the manipulation of attentional deployment, as an emotion regulation strategy, which involves a shift in attention away from the emotional aspects of emotion eliciting events by engaging in a competing task (Gross, 2008), or by changing the focus of the recollected memories, as it is the case in the present study.

In summary, the present findings suggest that the IFC's involvement during the recollection of emotional AMs is susceptible to manipulations of the retrieval focus only in the case of positive AMs, and that this region plays a role in both the enhancement and the inhibition of emotional experience during AM recollection. These findings have direct relevance for therapeutic interventions in affective disorders by pointing to the fact that the increased effectiveness of positive AMs in triggering strong emotional responses, and therefore in alleviating negative mood, occurs only when specific re-experiencing of positive emotions is explicitly emphasized during autobiographical recollection.

\section{ACKNOWLEDGMENTS}

This research was supported by funds from NARSAD (currently the Brain \& Behavior Research Foundation), CPRF (currently Healthy Minds Canada), and the University of Illinois (to Florin Dolcos). Ekaterina Denkova was supported by a WyethCIHR Post-Doctoral Fellowship. The authors wish to thank Trisha Chakrabarty and Kristina Suen for assistance with data collection and analysis.

\section{REFERENCES}

Addis, D. R., Wong, A. T., and Schacter, D. L. (2007). Remembering the past and imagining the future: common and distinct neural substrates during event construction and elaboration. Neuropsychologia 45, 1363-1377. doi:10.1016/j. neuropsychologia.2006.10.016

Aron, A. R., Robbins, T. W., and Poldrack, R. A. (2004). Inhibition and the right inferior frontal cortex. Trends Cogn. Sci. 8, 170-177. doi:10.1016/j.tics.2004.02.010

Baciu, M. V., Rubin, C., Decorps, M. A., and Segebarth, C. M. (1999). fMRI assessment of hemispheric language dominance using a simple inner speech paradigm. NMR Biomed. 12, 293-298. doi:10.1002/(SICI)1099-1492(199908) 12:5<293::AID-NBM573>3.3.CO;2-Y

Badre, D. (2008). Cognitive control, hierarchy, and the rostro-caudal organization of the frontal lobes. Trends Cogn. Sci. 12, 193-200. doi:10.1016/j.tics.2008.02.004

Badre, D., and Wagner, A. D. (2007). Left ventrolateral prefrontal cortex and the cognitive control of memory. Neuropsychologia 45, 2883-2901. doi:10.1016/j. neuropsychologia.2007.06.015

Battig, W. F., and Montague, W. E. (1969). Category norms of verbal items in 56 categories A replication and extension of the connecticut category norms. J. Exp. Psychol. 80, 1-46. doi:10.1037/h0027577

Beer, J. S., Knight, R. T., and D'Esposito, M. (2006). Controlling the integration of emotion and cognition: the role of frontal cortex in distinguishing helpful from hurtful emotional information. Psychol. Sci. 17, 448-453. doi:10.1111/j.1467-9280.2006.01726.x

Brand, M., and Markowitsch, H. J. (2006). "Memory processes and the orbitofrontal cortex," in The Orbitofrontal Cortex, eds D. H. Zald and S. L. Rauch (Oxford: Oxford University Press), 285-306.

Brand, M., and Markowitsch, H. J. (2008). "The role of the prefrontal cortex in episodic memory," in Handbook of Episodic Memory (Handbook of behavioral neuroscience, Vol. 18), eds E. Dere, J. P. Huston, and A. Easton (Amsterdam: Elsevier), 317-341.

Brewin, C. R., Reynolds, M., and Tata, P. (1999). Autobiographical memory processes and the course of depression. J. Abnorm. Psychol. 108, 511-517. doi:10.1037/0021-843X.108.3.511

Chang, L. J., Yarkoni, T., Khaw, M. W., and Sanfey, A. G. (2012). Decoding the role of the insula in human cognition: functional parcellation and large-scale reverse inference. Cereb. Cortex 23, 739-749. doi:10.1093/cercor/bhs065

Conway, M. A., and Pleydell-Pearce, C. W. (2000). The construction of autobiographical memories in the self-memory system. Psychol. Rev. 107, 261-288. doi:10.1037/0033-295X.107.2.261

Conway, M. A., Turk, D. J., Miller, S. L., Logan, J., Nebes, R. D., Meltzer, C. C., et al. (1999). A positron emission tomography (PET) study of autobiographical memory retrieval. Memory 7, 679-702. doi:10.1080/096582199387805

Craig, A. D. (2009). How do you feel - now? The anterior insula and human awareness. Nat. Rev. Neurosci. 10, 59-70. doi:10.1038/nrn2555

Dalgleish, T., Navrady, L., Bird, E., Hill, E., Dunn, B. D., and Golden, A. M. (2013). Method-of-loci as a mnemonic device to facilitate access to selfaffirming personal memories for individuals with depression. Clin. Psychol. Sci. doi:10.1177/2167702612468111

D'Argembeau, A., and Van der Linden, M. (2008). Remembering pride and shame: self-enhancement and the phenomenology of autobiographical memory. Memory 16, 538-547. doi:10.1080/09658210802010463

Daselaar, S. M., Rice, H. J., Greenberg, D. L., Cabeza, R., LaBar, K. S., and Rubin, D. C. (2008). The spatiotemporal dynamics of autobiographical memory: neural correlates of recall, emotional intensity, and reliving. Cereb. Cortex 18, 217-229. doi:10.1093/cercor/bhm048

Denkova, E., Chakrabarty, T., Dolcos, S., and Dolcos, F. (2011). Brain imaging investigation of the neural correlates of emotional autobiographical recollection. $J$. Vis. Exp. 54, p. ii:2396. doi:10.3791/2396

Denkova, E., Dolcos, S., and Dolcos, F. (2012). Reliving emotional personal memories: affective biases linked to personality and sex-related differences. Emotion 12, 515-528. doi:10.1037/a0026809

Denkova, E., Dolcos, S., and Dolcos, F. (2013). The effect of the retrieval focus and valence on the medial temporal lobe activity during autobiographical recollections. Front. Behav. Neurosci. 7:109. doi:10.3389/fnbeh.2013.00109

Depue, B. E., Curran, T., and Banich, M. T. (2007). Prefrontal regions orchestrate suppression of emotional memories via a two-phase process. Science 317, 215-219. doi:10.1126/science. 1139560

Diener, E., and Seligman, M. E. (2002). Very happy people. Psychol. Sci. 13, 81-84. doi:10.1111/1467-9280.00415

Dolcos, F., Diaz-Granados, P., Wang, L., and McCarthy, G. (2008). Opposing influences of emotional and non-emotional distracters upon sustained prefrontal cortex activity during a delayed-response working memory task. Neuropsychologia 46, 326-335. doi:10.1016/j.neuropsychologia.2007.07.010

Dolcos, F., Iordan, A. D., and Dolcos, S. (2011). Neural correlates of emotioncognition interactions: a review of evidence from brain imaging investigations. J. Cogn. Psychol. (Hove) 23, 669-694. doi:10.1080/20445911.2011.594433

Dolcos, F., Iordan, A. D., Kragel, J., Stokes, J., Campbell, R., McCarthy, G., et al. (2013). Neural correlates of opposing effects of emotional distraction on working 
memory and episodic memory: an event-related FMRI investigation. Front. Psychol. 4:293. doi:10.3389/fpsyg.2013.00293

Dolcos, F., Kragel, P., Wang, L., and McCarthy, G. (2006). Role of the inferior frontal cortex in coping with distracting emotions. Neuroreport 17, 1591-1594. doi:10.1097/01.wnr.0000236860.24081.be

Dolcos, F., LaBar, K. S., and Cabeza, R. (2004). Dissociable effects of arousal and valence on prefrontal activity indexing emotional evaluation and subsequent memory: an event-related fMRI study. Neuroimage 23, 64-74. doi:10.1016/j. neuroimage.2004.05.015

Fletcher, P. C., and Henson, R. N. (2001). Frontal lobes and human memory: insights from functional neuroimaging. Brain 124(Pt 5), 849-881. doi:10.1093/brain/ 124.5.849

Fredrickson, B. L. (2004). The broaden-and-build theory of positive emotions. Philos. Trans. R. Soc. Lond. B Biol. Sci. 359, 1367-1378. doi:10.1098/rstb.2004.1512

Fuster, J. M. (2002). Frontal lobe and cognitive development. J. Neurocytol. 31, 373-385. doi:10.1023/A:1024190429920

Greenberg, D. L., Rice, H. J., Cooper, J. J., Cabeza, R., Rubin, D. C., and Labar, K. S. (2005). Co-activation of the amygdala, hippocampus and inferior frontal gyrus during autobiographical memory retrieval. Neuropsychologia 43, 659-674. doi:10.1016/j.neuropsychologia.2004.09.002

Grindrod, C. M., Bilenko, N. Y., Myers, E. B., and Blumstein, S. E. (2008). The role of the left inferior frontal gyrus in implicit semantic competition and selection: an event-related fMRI study. Brain Res. 1229, 167-178. doi:10.1016/j.brainres. 2008.07.017

Gross, J. J. (2008). "Emotion regulation," in Handbook of Emotions, eds M. Lewis, J. M. Haviland-Jones, and L. F. Barrett (New York, NY: Guilford), 497-512.

Hagoort, P. (2005). On Broca, brain, and binding: a new framework. Trends Cogn. Sci. 9, 416-423. doi:10.1016/j.tics.2005.07.004

Hooker, C. I., and Knight, R. T. (2006). "Role of the orbitofrontal cortex in the inhibition of emotion," in The Orbitofrontal Cortex, eds D. H. Zald and S. L. Rauch (New York: Oxford University Press), 307-324.

Iordan, A., Dolcos, S., and Dolcos, F. (2013). Neural signatures of the response to emotional distraction: a review of evidence from brain imaging investigations. Front. Hum. Neurosci 7:200. doi:10.3389/fnhum.2013.00200

Joormann, J., Siemer, M., and Gotlib, I. H. (2007). Mood regulation in depression: differential effects of distraction and recall of happy memories on sad mood. $J$. Abnorm. Psychol. 116, 484-490. doi:10.1037/0021-843X.116.3.484

Kelly, C., Toro, R., Di Martino, A., Cox, C. L., Bellec, P., Castellanos, F. X., et al. (2012).A convergent functional architecture of the insula emerges across imaging modalities. Neuroimage 61, 1129-1142. doi:10.1016/j.neuroimage.2012.03.021

Kim, S. H., and Hamann, S. (2007). Neural correlates of positive and negative emotion regulation. J. Cogn. Neurosci. 19, 776-798. doi:10.1162/jocn.2007.19.5.776

Levine, L. J., and Edelstein, R. S. (2009). Emotion and memory narrowing: a review and goal-relevance approach. Cogn. Emot. 23, 833-875. doi:10.1080/ 02699930902738863

Lindquist, K. A., Wager, T. D., Kober, H., Bliss-Moreau, E., and Barrett, L. F. (2012). The brain basis of emotion: a meta-analytic review. Behav. Brain Sci. 35, 121-143. doi:10.1017/S0140525X11000446

Markowitsch, H. J. (1995). Which brain regions are critically involved in the retrieval of old episodic memory? Brain Res. Brain Res. Rev. 21, 117-127. doi:10.1016/0165-0173(95)00007-0

Markowitsch, H. J., Thiel, A., Reinkemeier, M., Kessler, J., Koyuncu, A., and Heiss, W. D. (2000). Right amygdalar and temporofrontal activation during autobiographic, but not during fictitious memory retrieval. Behav. Neurol. 12, 181-190.

Markowitsch, H. J., Vandekerckhove, M. M., Lanfermann, H., and Russ, M. O. (2003). Engagement of lateral and medial prefrontal areas in the ecphory of sad and happy autobiographical memories. Cortex 39, 643-665. doi:10.1016/S00109452(08)70858-X

McGuire, P. K., Silbersweig, D. A., Murray, R. M., David, A. S., Frackowiak, R. S., and Frith, C. D. (1996). Functional anatomy of inner speech and auditory verbal imagery. Psychol. Med. 26, 29-38. doi:10.1017/S0033291700033699

Morin, A., and Michaud, J. (2007). Self-awareness and the left inferior frontal gyrus: inner speech use during self-related processing. Brain Res. Bull. 74, 387-396. doi:10.1016/j.brainresbull.2007.06.013

Moss, H. E., Abdallah, S., Fletcher, P., Bright, P., Pilgrim, L., Acres, K., et al. (2005). Selecting among competing alternatives: selection and retrieval in the left inferior frontal gyrus. Cereb. Cortex 15, 1723-1735. doi:10.1093/cercor/bhi049

Ochsner, K. N., Ray, R. D., Cooper, J. C., Robertson, E. R., Chopra, S., Gabrieli, J. D., et al. (2004). For better or for worse: neural systems supporting the cognitive down- and up-regulation of negative emotion. Neuroimage 23, 483-499. doi:10. 1016/j.neuroimage.2004.06.030

Ochsner, K. N., Silvers, J. A., and Buhle, J. T. (2012). Functional imaging studies of emotion regulation: a synthetic review and evolving model of the cognitive control of emotion. Ann. N. Y. Acad. Sci. 1251, E1-E24. doi:10.1111/j.1749-6632. 2012.06751.x

Petrides, M., and Pandya, D. N. (2002). Comparative cytoarchitectonic analysis of the human and the macaque ventrolateral prefrontal cortex and corticocortical connection patterns in the monkey. Eur. J. Neurosci. 16, 291-310. doi:10.1046/j.1460-9568.2001.02090.x

Piolino, P., Giffard-Quillon, G., Desgranges, B., Chetelat, G., Baron, J. C., and Eustache, F. (2004). Re-experiencing old memories via hippocampus: a PET study of autobiographical memory. Neuroimage 22, 1371-1383. doi:10.1016/j. neuroimage.2004.02.025

Poldrack, R. A., Wagner, A. D., Prull, M. W., Desmond, J. E., Glover, G. H., and Gabrieli, J. D. (1999). Functional specialization for semantic and phonological processing in the left inferior prefrontal cortex. Neuroimage 10, 15-35. doi:10.1006/nimg.1999.0441

Rubin, D. C., Berntsen, D., and Bohni, M. K. (2008). A memory-based model of posttraumatic stress disorder: evaluating basic assumptions underlying the PTSD diagnosis. Psychol. Rev. 115, 985-1011. doi:10.1037/a0013397

Salas, C. E., Radovic, D., and Turnbull, O. H. (2012). Inside-out: comparing internally generated and externally generated basic emotions. Emotion 12, 568-578. doi:10.1037/a0025811

Svoboda, E., McKinnon, M. C., and Levine, B. (2006). The functional neuroanatomy of autobiographical memory: a meta-analysis. Neuropsychologia 44, 2189-2208. doi:10.1016/j.neuropsychologia.2006.05.023

Thompson-Schill, S. L., D’Esposito, M., Aguirre, G. K., and Farah, M. J. (1997). Role of left inferior prefrontal cortex in retrieval of semantic knowledge: a reevaluation. Proc. Natl. Acad. Sci. U.S.A. 94, 14792-14797. doi:10.1073/pnas.94.26.14792

Thompson-Schill, S. L., D’Esposito, M., and Kan, I. P. (1999). Effects of repetition and competition on activity in left prefrontal cortex during word generation. Neuron 23, 513-522. doi:10.1016/S0896-6273(00)80804- 1

Uddin, L. Q., Kinnison, J., Pessoa, L., and Anderson, M. L. (2013). Beyond the tripartite cognition-emotion-interoception model of the human insular cortex. J. Cogn. Neurosci. 26, 16-27. doi:10.1162/jocn_a_00462

Wager, T. D., Barrett, L. F., Bliss-Moreau, E., Lindquist, K., Duncan, S., Kober, H., et al. (2008). "The neuroimaging of emotion," in Handbook of Emotion, eds M. Lewis, J. M. Haviland-Jones, and L. F. Barrett (New York: Guilford), 249-271.

Werner-Seidler, A., and Moulds, M. L. (2011). Autobiographical memory characteristics in depression vulnerability: formerly depressed individuals recall less vivid positive memories. Cogn. Emot. 25, 1087-1103. doi:10.1080/02699931. 2010.531007

Werner-Seidler, A., and Moulds, M. L. (2012). Mood repair and processing mode in depression. Emotion 12, 470-478. doi:10.1037/a0025984

Young, K. D., Bellgowan, P. S., Bodurka, J., and Drevets, W. C. (2013). Functional neuroimaging of sex differences in autobiographical memory recall. Hum. Brain Mapp. 34, 3320-3332. doi:10.1002/hbm.22144

Zaki, J., Davis, J. I., and Ochsner, K. N. (2012). Overlapping activity in anterior insula during interoception and emotional experience. Neuroimage 62, 493-499. doi:10.1016/j.neuroimage.2012.05.012

Conflict of Interest Statement: The authors declare that the research was conducted in the absence of any commercial or financial relationships that could be construed as a potential conflict of interest.

Received: 15 October 2013; paper pending published: 02 November 2013; accepted: 19 November 2013; published online: 16 December 2013.

Citation: Denkova E, Dolcos S and Dolcos F (2013) The effect of retrieval focus and emotional valence on the inferior frontal cortex activity during autobiographical recollection. Front. Behav. Neurosci. 7:192. doi: 10.3389/fnbeh.2013.00192

This article was submitted to the journal Frontiers in Behavioral Neuroscience.

Copyright (c) 2013 Denkova, Dolcos and Dolcos. This is an open-access article distributed under the terms of the Creative Commons Attribution License (CC BY). The use, distribution or reproduction in other forums is permitted, provided the original author(s) or licensor are credited and that the original publication in this journal is cited, in accordance with accepted academic practice. No use, distribution or reproduction is permitted which does not comply with these terms. 